\title{
TNKS1BP1 functions in DNA double-strand break repair though facilitating DNA-PKCS autophosphorylation dependent on PARP-1
}

\author{
Lian-Hong Zou ${ }^{1,2}$, Zeng-Fu Shang ${ }^{2,3}$, Wei Tan ${ }^{1,2}$, Xiao-Dan Liu ${ }^{2}$, Qin-Zhi $\mathbf{X u}^{2}$, \\ Man Song ${ }^{2,3}$, Yu Wang ${ }^{2}$, Hua Guan ${ }^{2}$, Shi-Meng Zhang ${ }^{2}$, Lan Yu${ }^{4}$, Cai-Gao Zhong ${ }^{1}$, \\ Ping-Kun Zhou ${ }^{2,3}$ \\ ${ }^{1}$ School of Public Heath, Central South University, Changsha, Hunan Province 410078, P. R. China \\ ${ }^{2}$ Department of Radiation Toxicology and Oncology, Beijing Key Laboratory for Radiobiology, Beijing Institute of Radiation \\ Medicine, Beijing 100850, P. R. China \\ ${ }^{3}$ School of Radiation Medicine and Protection, Medical College of Soochow University, Collaborative Innovation Center of \\ Radiation Medicine of Jiangsu Higher Education Institutions, Suzhou 215123, China \\ ${ }^{4}$ Department of Radiation Oncology, University of Texas Southwestern Medical Center, Dallas, TX 75390, USA
}

Correspondence to:

Ping-Kun Zhou, e-mail: zhoupk@bmi.ac.cn

Zeng-Fu Shang, e-mail: zengfu.shang@suda.edu.cn

Keywords: TNKS1BP1, radiation, DNA-PKCS, DNA repair, poly(ADP-ribosyl)ation

Received: December 14, $2014 \quad$ Accepted: January 10, $2015 \quad$ Published: February 04, 2015

\section{ABSTRACT}

TNKS1BP1 was originally identified as an interaction protein of tankyrase 1, which belongs to the poly(ADP-ribose) polymerase (PARP) superfamily. PARP members play important roles for example in DNA repair, telomere stability and mitosis regulation. Although the TNKS1BP1 protein was considered to be a poly(ADPribosyl)ation acceptor of tankyrase 1, its function is still unknown. Here we firstly identified that TNKS1BP1 was up-regulated by ionizing radiation (IR) and the depletion of TNKS1BP1 significantly sensitized cancer cells to IR. Neutral comet assay, pulsed-field gel electrophoresis, and YH2AX foci analysis indicated that TNKS1BP1 is required for the efficient repair of DNA double-strand breaks (DSB). The TNKS1BP1 protein was demonstrated to interact with DNA-dependent protein kinase (DNA-PKcs) and poly(ADP-ribose) polymerase 1 (PARP-1), by co-immunoprecipitation analysis. Moreover, TNKS1BP1 was shown to promote the association of PARP-1 and DNAPKcs. Overexpression of TNKS1BP1 induced the autophosphorylation of DNA-PKcs/ Ser2056 in a PARP-1 dependent manner, which contributed to an increased capability of DNA DSB repair. Inhibition of PARP-1 blocked the TNKS1BP1-mediated DNA-PKCS autophosphorylation and attenuated the PARylation of DNA-PKCs. TNKS1BP1 is a newly described component of the DNA DSB repair machinery, which provides much more mechanistic evidence for the rationale of developing effective anticancer measures by targeting PARP-1 and DNA-PKcs.

\section{INTRODUCTION}

DNA double-strand breakage (DSB) induced by either ionizing radiation or genotoxic chemicals is a particularly hazardous type of DNA damage to dividing cells, because it involves a break in both DNA strands nearby in the double helix. There are two major interconnected and collaborative pathways to repair this damage: homologous recombination (HR) and non-homologous end joining (NHEJ) [1]. Due to needing a homologous template to guide repair of the broken DNA strand, the HR repair pathway is believed to be activated after DNA replication in the late $\mathrm{S}$ and $\mathrm{G} 2$ phases of the cell cycle. Whereas NHEJ repair could mediate the direct re-ligation of the broken DNA molecule, this type of repair does not require a homologous template and is not restricted to a particular phase of the cell cycle. Thus, NHEJ is thought to be the prevailing pathway for DSB 
repair [2]. The DNA dependent protein kinase complex (DNA-PK) is a critical component of the NHEJ pathway, which consists of a Ku70/Ku80 heterodimer and the DNA dependent protein kinase catalytic subunit (DNA-PKcs). DNA-PK plays an essential role in the pathway of NHEJ repair, including initiating DSB recognition, regulating access to breaks, promoting repair machinery assembly and DNA molecule ligation [3, 4]. As the catalytic subunit, DNA-PKcs is a member of the phosphatidylinositol-3 (PI-3) kinase-like family (PI3KK), and it is recruited to DNA damage sites by the Ku70/Ku86 heterodimer $[5,6]$. The activation of DNA-PKcs in response to DNA damage is dependent on its autophosphorylation within two cluster sites (ABCDE and PQR) $[4,7]$ and/ or phosphorylated by ATM [8]. The activated DNA-PKcs can phosphorylate a series of canonical repair factors in the NHEJ pathway, including Ku70/Ku86, Artemis, XRCC4, DNA ligase IV, XLF, Werner (WRN) and other DNA damage response proteins: including polynucleotide kinase/phosphatase (PNKP), histone H2AX, P53, CHK2, AKT $[3,9]$. However, how the recruitment of DNAPKcs onto DNA lesion sites stimulates its activity, or how its autophosphorylation is initiated, has not been fully clarified. It is particularly worthwhile to identify the potential proteins or other types of post-translational modifications which may modulate the DNA-PKcs activity and hence play a role in DNA double-strand break repair.

Poly(ADP-ribosyl)ation (PARylation), which is catalyzed by Poly(ADP-ribose) polymerases (PARP), recently has become an increasingly interested subject regarding protein post-translational modifications in the research field of DNA damage response and cancer therapeutic target exploration $[10,11]$. PARP transfers negatively charged ADP-ribose groups from donor $\mathrm{NAD}^{+}$ molecules onto their target proteins, a process named poly(ADP-ribosyl)ation. At least three PARP molecules, PARP-1, PARP-2 and PARP-3, have been identified to be activated dependently of DNA in the cellular response to DNA double-strand breaks [11]. PARP-1, which mediates about $85 \%$ of cellular poly-ADP-ribose (PAR) synthesis activity, plays a key role in DNA repair, including in HR, NHEJ, single-strand break repair and base excision repair [12]. Following DNA damage, PARP-1 quickly relocates to DNA damage sites and catalyzes protein PARylation $[13,14]$, which contributes to the recruitment of other DNA damage response factors onto DNA lesions [1520]. Inhibition or depletion of PARP-1 leads to a DNA DSBs NHEJ repair defect in hamster and murine cells after ionizing radiation (IR), manifesting in prolonged existence of the phosphorylated form of $\gamma \mathrm{H} 2 \mathrm{AX}$ foci which represents the persistence of DSBs [21-23]. Previous studies showed that PARP-1 could interact with DNA-PKcs and Ku70/Ku80 heterodimer [23-25]. PARP-1 can be phosphorylated by DNA-PKcs, and in turn, PARP-1 ADP-ribosylates the DNA-PKcs and increases the activity of DNA-PKcs. Interestingly, PARP-1 mediated
ADP-ribosylation and activation of DNA-PKcs does not rely on $\mathrm{Ku} 70 / 86$ heterodimers [24, 26, 27]. Hence, the molecular mechanism of how PARP-1 facilitates DNAPKcs activation remains to be elucidated.

Tankyrase 1 (TNKS1), the TRF1-interacting ankyrinrelated ADP-ribose polymerase, is a member of the PARP superfamily. Tankyrase 1 was found to play an important role in DNA repair, telomere length maintenance and mitotic progression regulation [28, 29], and has also been considered to be a potential target for cancer therapy [30, 31]. Previously, a tankyrase 1 binding protein 1 of $182 \mathrm{kDa}$ (TNKS1BP1, also named TAB182) has been identified in the co-immunoprecipitates of Tankyrase 1 [32]. TNKS1BP1 resides in both the nucleus and the cytoplasm, and displays a complex pattern of subcellular localization with Tankyrase 1. TNKS1BP1 was assumed to be poly ADP-ribosylated by Tankyrase $1[32,33]$. However, the biological function of TAB182 is still unknown. In this study, we report that the expression of TNKS1BP1 was increased by ionizing radiation and depletion of TNKS1BP1 significantly sensitized multiple cancer cell lines to $\gamma$-irradiation. Moreover, depletion of TNKS1BP1 significantly decreased the efficiency of repair of DNA double-strand breaks. TNKS1BP1 was found to interact directly with DNA-PKcs, $\mathrm{Ku} 70, \mathrm{Ku} 86$, and PARP-1. Overexpression of TNKS1BP1 promoted the interaction between PARP-1 and DNA-PKcs, and subsequently increased the phosphorylation of DNAPKcs /ser2056. Furthermore, inhibition of PARP-1 activity blocked the TNKS1BP1 mediated DNA-PKcs activation. These results implied that TNKS1BP1 is a novel modulator of radiation-induced DNA-PKcs phosphorylation, and contributes to DNA double-strand break repair through facilitating PARP-1/DNA-PKcs interaction. Thus, the TNKS1BP1 may represent a therapeutic target to improve the effect of radiotherapy.

\section{RESULTS}

\section{The contribution of TNKS1BP1 to the survival of irradiated cells}

We firstly investigated whether TNKS1BP1 was involved in the IR-induced DNA damage response. Growing HeLa cells were irradiated with 4 Gy $\gamma$-rays, and harvested at different time points post irradiation. Immunoblotting analysis revealed that the expression level of TNKS1BP1 increased at least as early as $2 \mathrm{~h}$ after irradiation. The inducible expression of TNKS1BP1 by ionizing irradiation reached a peak level at about $4-8$ $\mathrm{h}$ (Figure 1A). To test whether the IR-induced expression occurred in different cell lines, we gave human breast cancer MCF-7 cells, human hepatocellular cancer HepG2 cells and human normal liver L02 cells different doses of $\gamma$-rays. Levels of TNKS1BP1 protein in cell lysates were detected by immunoblotting analysis at $4 \mathrm{~h}$ after irradiation. An increased expression of TNKS1BP1 
was observed in these three cell lines after irradiation (Figure 1B). As TNKS1BP1 has been reported to localize in both cytoplasm and nucleus, we studied which part mainly contributes to IR-induced TNKS1BP1 overexpression by fractionating nucleus and cytoplasm and detecting the respective expression levels of TNKS1BP1. As shown in Figure 1C, the expression of TNKS1BP1 was increased in both the cytoplasm and nuclear fractions. We further performed a real-time PCR experiment and found that the mRNA levels of TNKS1BP1 were significantly increased in 4 Gy $\gamma$-irradiated HeLa cells (Figure 1D).

As the expression of TNKS1BP1 was upregulated in the irradiated cells, we further evaluated the role of
TNKS1BP1 in determining the cellular radiosensitivity. For the above purpose, we established the $T N K S 1 B P 1$ stably-knocked-down cell lines HeLa-shTNKS1BP1 (Figure 1E) and HepG2-shTNKS1BP1 (Figure 1F) through sustained expression of shRNA against TNKS1BP1 in HeLa cells and HepG2 cells, respectively. As shown in Figure $1 \mathrm{G}$ and $1 \mathrm{H}$, knockdown of TNKS1BP1 markedly decreased the survival rates of both HeLa cells (Figure $1 \mathrm{G})$ and HepG2 cells (Figure 1H) when compared to the control HeLa-NC and HepG2-NC cells after IR, respectively. These results demonstrated that TNKS1BP1 plays an important role in maintaining the surviving capability of cells against IR.

A

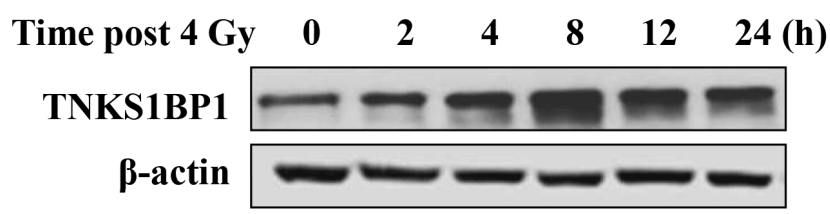

B

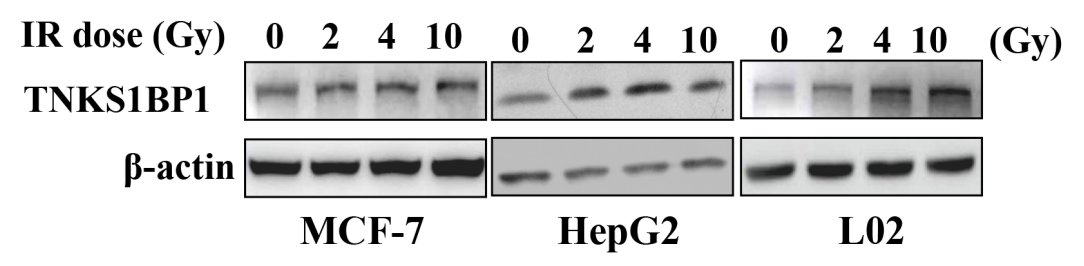

D
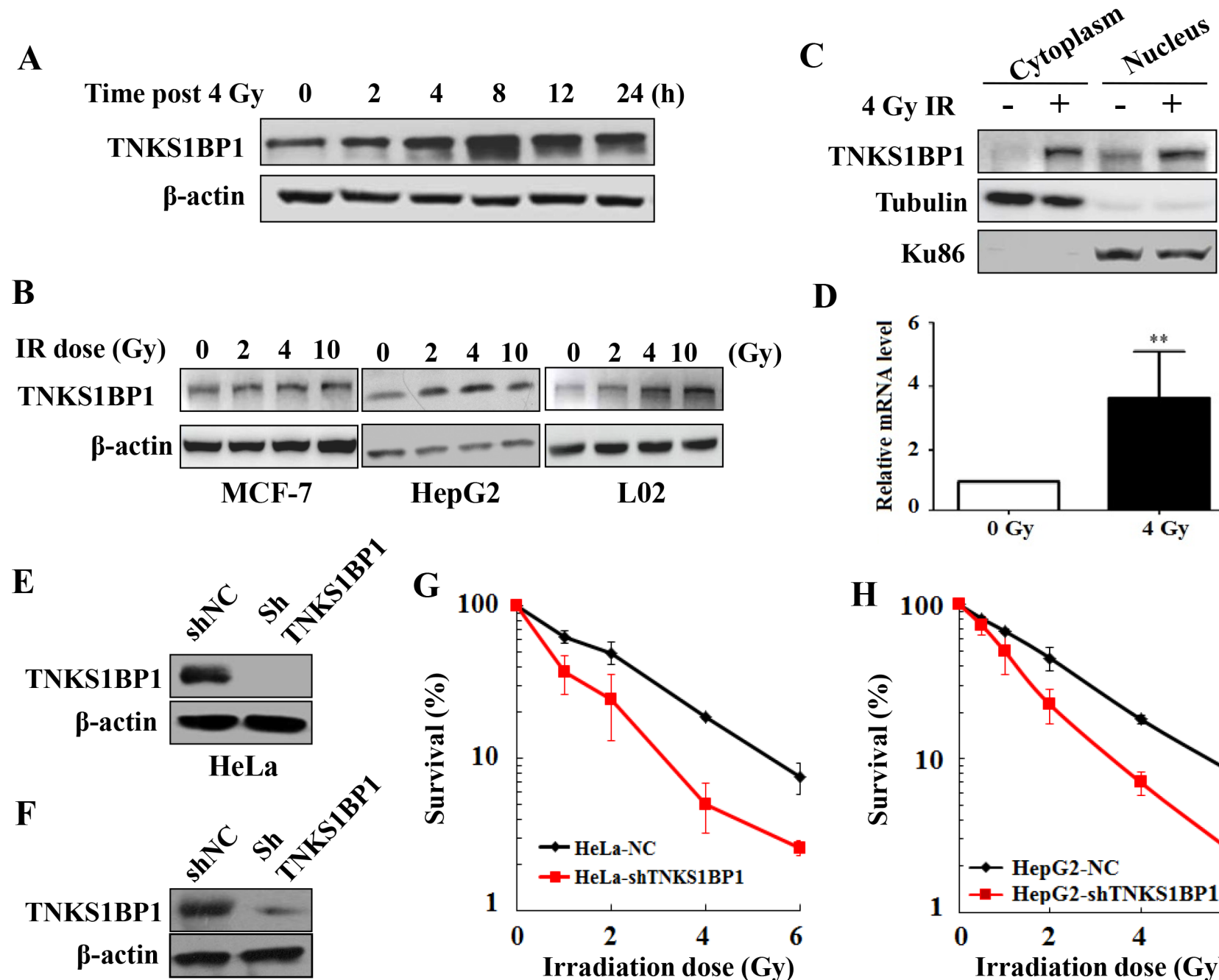

HepG2
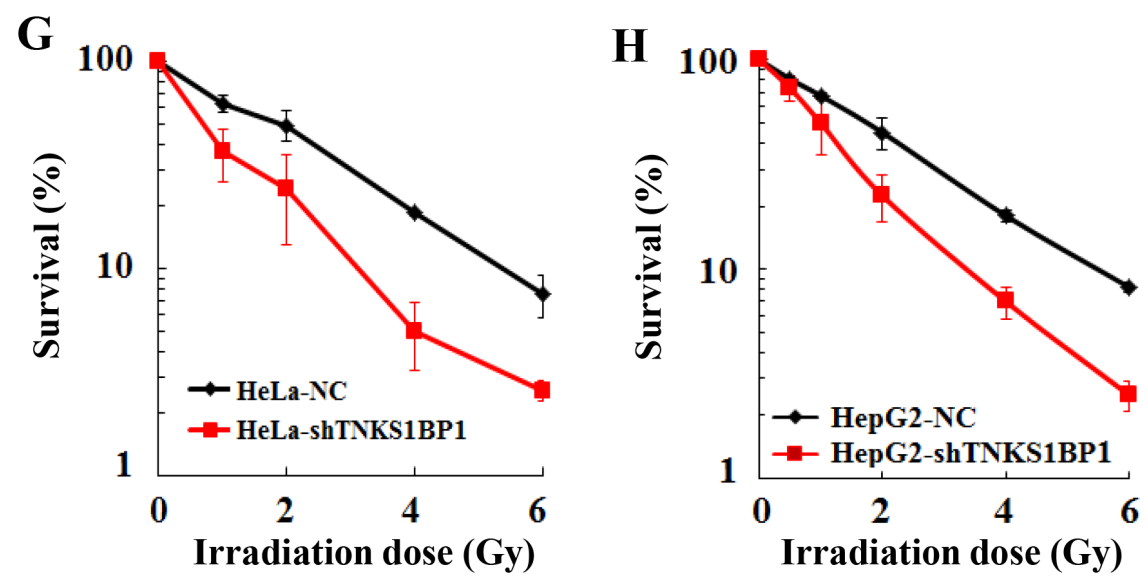

Figure 1: Upregulation of TNKS1BP1 by ionizing radiation (IR) and its effect on the radiosensitivity of cells. (A) Immunoblotting hybridization showing the increased expression of TNKS1BP1 protein in HeLa cells at given times after exposed to 4 Gy of $\gamma$-rays. (B) Immunoblotting hybridization showing the increased expression of TNKS1BP1 protein in MCF7, HepG2 and L02 cells at $4 \mathrm{~h}$ after different doses of $\gamma$-rays. (C) Increased TNKS1BP1 protein levels in both cytoplasm and nuclei in HeLa cells at $4 \mathrm{~h}$ after 4 Gy of $\gamma$-rays. (D) Quantitative real-time RT-PCR analysis showing the increased mRNA expression of TNKS1BP1 in HeLa cells at 2 $\mathrm{h}$ after 4 Gy of $\gamma$-rays. (E) Depletion of TNKS1BP1 expression in HeLa cells mediated by specific shRNA. (F) Depletion of TNKS1BP1 expression in HepG2 cells mediated by specific shRNA. (G) TNKS1BP1 depleted HeLa-shTNKS1BP1 cells became much more sensitive to IR as compared to the control HeLa-NC cells. (H) TNKS1BP1 depleted HepG2-shTNKS1BP1 cells became much more sensitive to IR as compared to the control HepG2-NC cells. 
TNKS1BP1 plays a role in DNA double-strand break repair

Decreased DNA DSB repair is an important mechanism by which cells become more sensitive to IR. To measure the repair kinetics of IR-induced DSBs, HeLa-NC and HeLa-shTNKS1BP1 cells were irradiated with $4 \mathrm{~Gy}$ and harvested at the indicated time points. The Immunofluorescence analysis showed that $\gamma \mathrm{H} 2 \mathrm{AX}$ foci after irradiation in TNKS1BP1 knockdown HeLa cells exhibited prolonged repair kinetics as compared to control cells (Figure 2A and 2B). To further confirm this defect in DSB repair capacity of HeLa-shTNKS1BP1 cells, the pulsed-field gel electrophoresis assay (Figure $2 \mathrm{C}$ and 2D) and neutral comet assay (Supplementary Figure $1 \mathrm{~A}$ and $1 \mathrm{~B}$ ) were performed to assess the repair efficiency of IR-induced DSBs. The results clearly indicated that the repair efficiency of IR-induced DSBs was significantly decreased in TNKS1BP1-suppresed HeLa cells.

The alteration of cell cycle progression is another critical cellular response to the DNA damage signal induced by IR. As shown in Supplementary Figure 2A and $2 \mathrm{~B}$, at $12 \mathrm{~h}$ post 4 Gy IR, HeLa-shTNKS1BP1 and $\mathrm{HeLa}-\mathrm{NC}$ cells were largely arrested at $\mathrm{G}_{2} / \mathrm{M}$ phase. At 24 h after 4 Gy IR, HeLa-NC cells almost completely recovered from the $\mathrm{G}_{2} / \mathrm{M}$ blockage. However, HeLashTNKS1BP1 cells still exhibited robust $\mathrm{G}_{2} / \mathrm{M}$ arrest $(50.6 \%)$ at the same time point. Furthermore, we investigated whether the shRNA-resistant TNKS1BP1 expressing vector could rescue this abnormal cell cycle progression phenotype in HeLa-shTNKS1BP1 cells. We found that the delayed recovery of $\mathrm{G}_{2} / \mathrm{M}$ arrest in HeLashTNKS1BP1 cells was rescued by stably overexpressing the shRNA-resistant Myc-TNKS1BP1 (Supplementary Figure 2C and 2D).
A

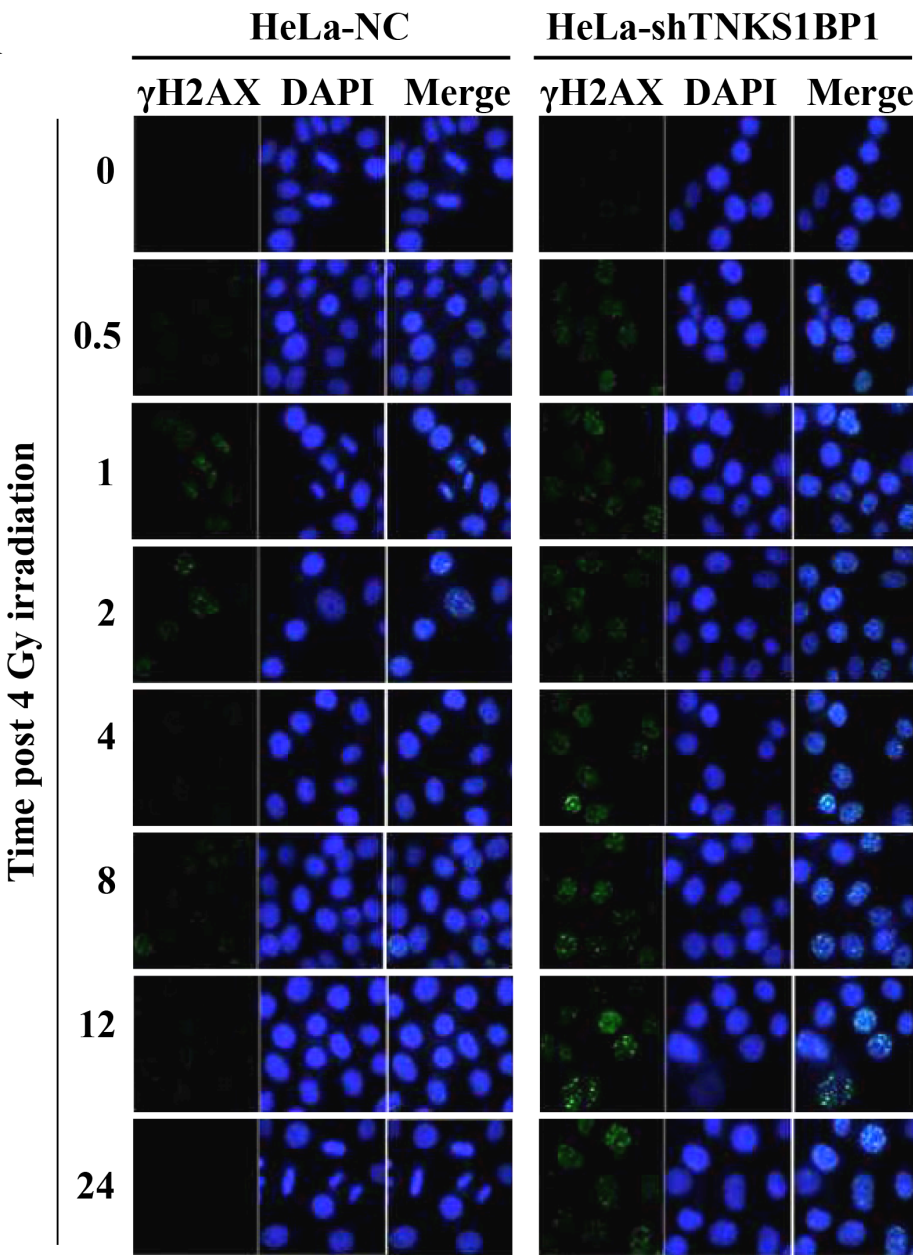

B

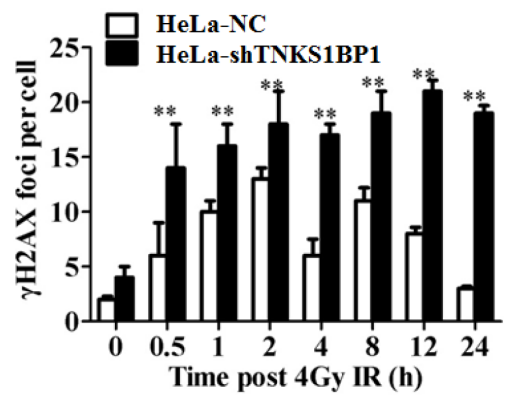

C

Time post 4Gy IR (h)

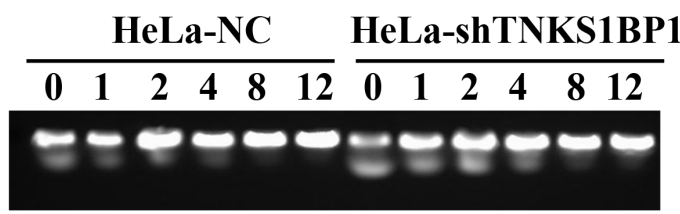

D

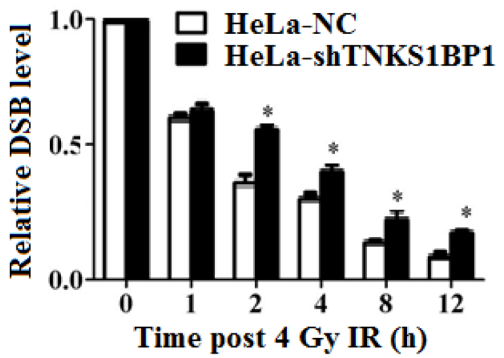

Figure 2: Depletion of TNKS1BP1 leads to defective DNA double-strand break repair. (A) Immunofluorescence staining image of $\gamma \mathrm{H} 2 \mathrm{AX}$ foci in TNKS1BP1 depleted HeLa-shTNKS1BP1 cells and control HeLa-NC cells at given times after 4 Gy of $\gamma$-rays. (B) Residual number of $\gamma \mathrm{H} 2 \mathrm{AX}$ foci per cell after certain times of repair in $4 \mathrm{~Gy}$ irradiated cells. ${ }^{*} P<0.01$. (C) Pulsed-gel electrophoresis (PFGE) pattern of DNA double-strand breaks and repair in 4 Gy-irradiated cells. (D) Repair kinetics of 4 Gy-induced DSBs detected by PFGE. $* P<0.05$. 


\section{TNKS1BP1 facilitates the phosphorylation of DNA-PKes at Ser2056}

Since TNKS1BP1 is involved in DNA damage response, and plays a role in DNA DSB repair, we investigated whether the depletion of TNKS1BP1 in HeLa cells would disturb the signaling transduction of the DNA damage response induced by IR. The Ser2056 has been considered as an autophosphorylation site of DNAPKcs, and its phosphorylation represents the activation of DNA-PKcs. Our result indicated that the phosphorylation of DNA-PKcs/Ser2056 was significantly impaired in TNKS1BP1-deficient HeLa cells (Figure 3A). DNAPKcs has been previously shown to phosphorylate Chk2 in response to DNA damage induced by IR or during normal mitosis progression [34-36]. As shown in Figure 3A, the IR-induced phosphorylation of Chk2 at T68 site was much weaker in TNKS1BP1 deficient HeLa cells compared to control cells, indicating that the deficiency of TNKS1BP1 disturbed the activation of the down-stream substrates of DNA-PKcs. Moreover, overexpression of TNKS1BP1 increased the phosphorylation of DNA-PKcs/Ser2056

A HeLa-NC HeLa-shTNKS1BP1

Time post 4Gy IR: $\begin{array}{lllllllllllll}0 & 1 & 2 & 4 & 8 & 12 & 0 & 1 & 2 & 4 & 8 & 12 & \text { (h) }\end{array}$ TNKS1BP1 PKes/pS2056

DNA-PKes Chk2 pT68

Chk2 $11717 \mathrm{~mm}$
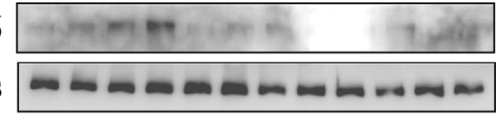

$\beta$-actin
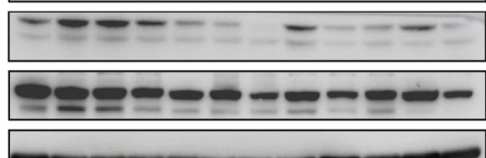

B

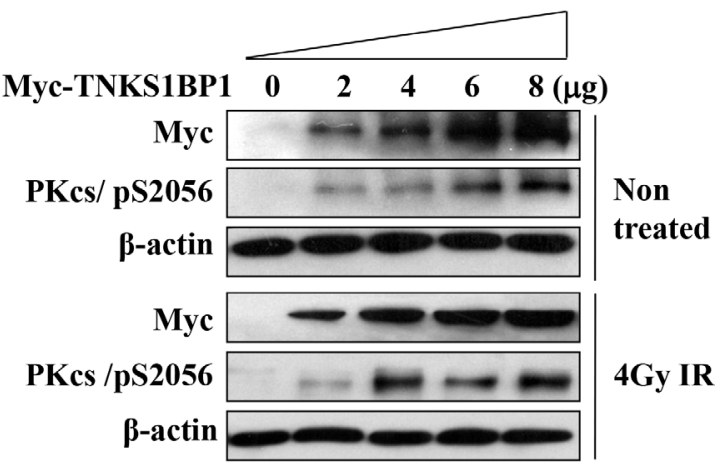

C

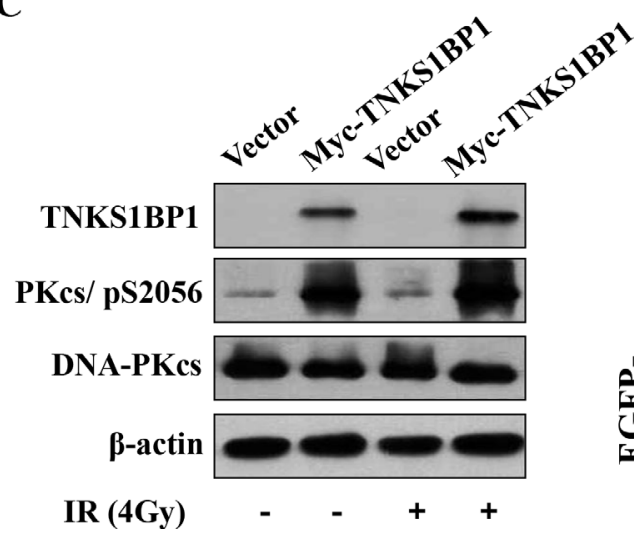

D
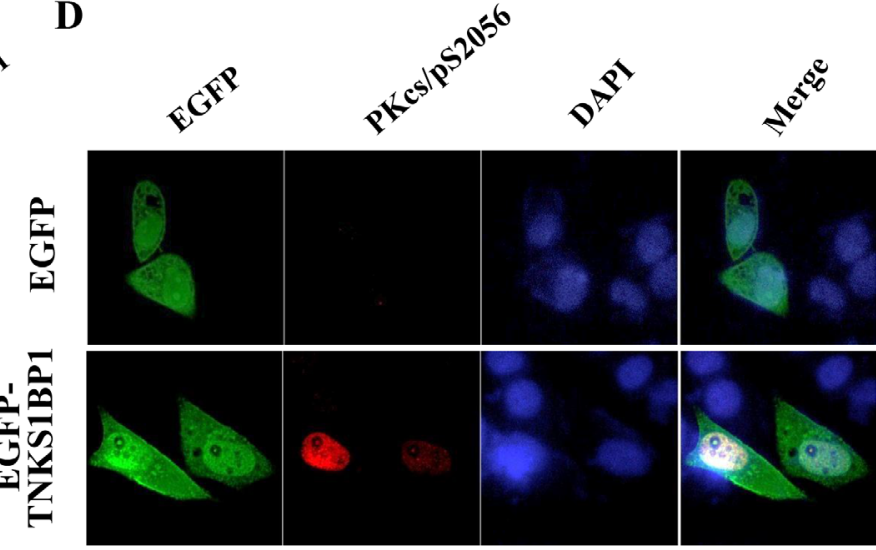

Figure 3: TNKS1BP1-medated autophosphorylation of DNA-PKes/S2056. (A) Immunoblotting hybridization showing that depletion of TNKS1BP1 blocked the autophosphorylation of DNA-PKcs/S2056 and phosphorylation of CHK2/T68 induced by IR in HeLa cells. (B) Overexpression of TNKS1BP1 induced the autophosphorylation of DNA-PKcs/S2056 in HEK-293 cells either with or without irradiation. (C) shRNA-resistant Myc-TNKS1BP1 increased the autophosphorylation level of DNA-PKcs/S2056 in TNKS1BP1-depleted HeLa-shTNKS1BP1 cells. (D) Immunofluorescence image showing the autophosphrylation of DNA-PKcs/S2056 in HeLa cells transfected and expressing EGFP-tagged TNKS1BP1 plasmid. 
was used to immunoprecipitate the TNKS1BP1-interacting complex from HeLa cell extracts. Western blotting analysis demonstrated that the DNA-PK complex (Ku70/ Ku80/DNA-PKcs) was detected in the immunoprecitates of TNKS1BP1 (Figure 4A). In addition, PARP-1 was also shown in the TNKS1BP1-interacted complex. Moreover, TNKS1BP1 and PARP-1 were detected in the complex immunoprecipitated by the antibody against DNA-PKcs (Figure 4B), and DNA-PKcs and TNKS1BP1 were found in the complex immunoprecipated by the antibody against PARP-1 (Figure 4C). Therefore, DNA-PK, TNKS1BP1 and PARP-1 could form a multiple complex in the DNA damage response.

The interaction between PARP-1 and DNA-PK has also been previously described in several independent studies [23-25]. In order to understand the effect of TNKS1BP1 on the interaction of DNA-PKcs and PARP1, we used the DNA-PKcs antibody to perform the immunoprecipitation assays from lysates of the cells with a control normal level of TNKS1BP1 (HeLa-NC), shRNAmediated depleted TNKS1BP1 (HeLa-shTNKS1BP1), and overexpressed TNKS1BP1 (HeLa-mycTNKS1BP1). As shown in Figure 4E, the association of PARP-1 with DNA-PKcs was weakened in HeLa-shTNKS1BP1 cells. Whereas, overexpressed TNKS1BP1did promote the association of PARP-1 with DNA-PKcs (Figure 4E). Therefore, TNKS1BP1 could play a role in mediating the association of DNA-PKcs and PARP-1.

\section{Activation of DNA-PKes mediated by TNKS1BP1 relies on the ADP-ribose polymerase activity of PARP-1}

PARP-1 has been suggested to PARylate DNAPKcs, and thereby to stimulate the kinase activity of DNA-PKcs [19, 21, 23, 24]. Here we also observed the PARylation modification of DNA-PKcs in the cells, which was attenuated by the PARP-1 inhibitor 3-AB
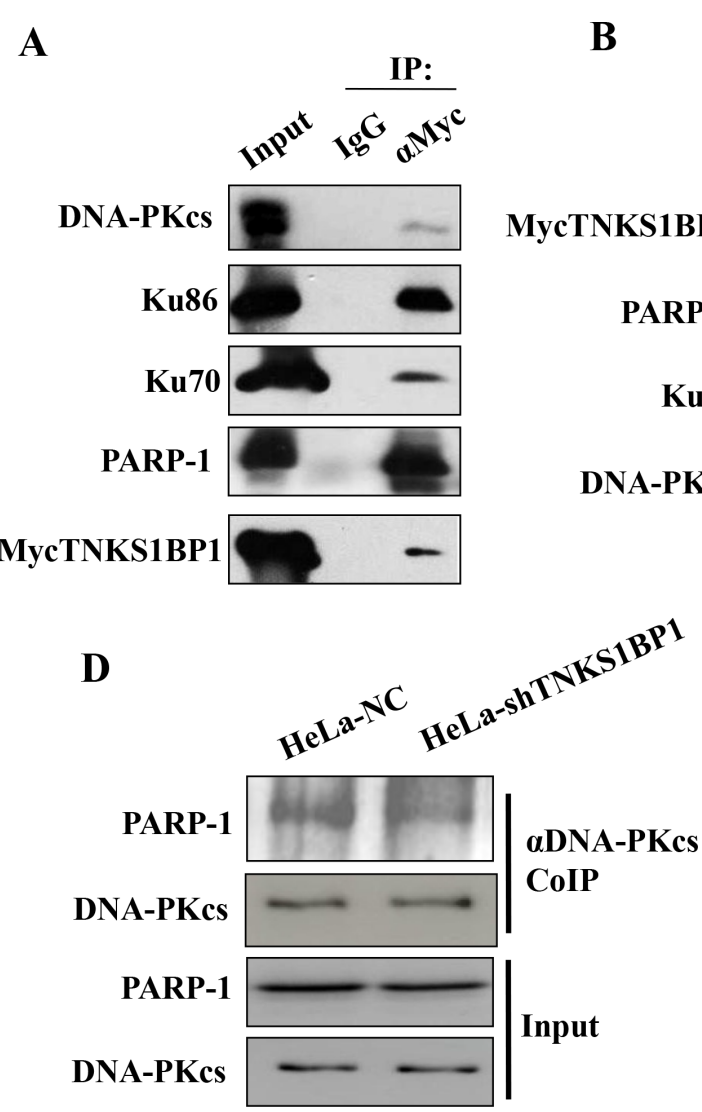

B

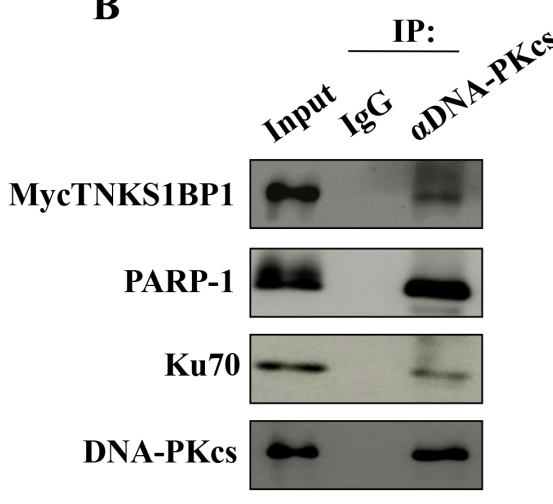

$\mathbf{E}$
C

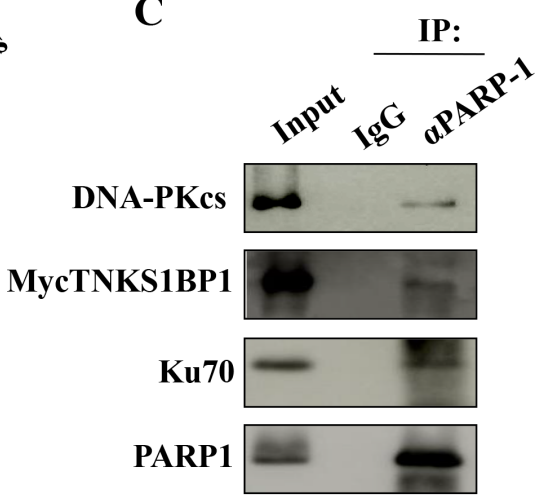

Figure 4: TNKS1BP1 mediated the interaction of DNA-PKcs and PARP-1. (A) Interaction of TNKS1BP1 with the DNAPK complex and PARP-1 was shown by co-immunoprecipitation (Co-IP) with antibody against myc from HeLa cells transfected with myc-tagged TNKS1BP1, or with IgG control. (B) Interaction of DNA-PKes with TNKS1BP1 and PARP-1 was shown by Co-IP with the antibody against DNA-PKcs from HeLa cells transfected with myc-tagged TNKS1BP1, or with IgG control. (C) Interaction of PARP-1 with TNKS1BP1 and DNA-PKcs was shown by Co-IP with the antibody against PARP-1 from HeLa cells transfected with myc-tagged TNKS1BP1, or with IgG control. (D) A decreased PARP-1 level in the co-immunoprecipitated complex of the antibody against DNA-PKcs in TNKS1BP1-depleted HeLa-shTNKS1BP1 cells as compared to that from control HeLa-NC cells. (E) Increased PARP-1 level in the co-immunoprecipitated complex of the antibody against DNA-PKcs in TNKS1BP1-overexpressed HeLa-mycTNKS1BP1 cells as compared to that from control HeLa-NC cells. 
(Figure 5A). To test whether TNKS1BP1-mediated activation of DNA-PKcs relies on the activity of PARP-1, we treated TNSKS1BP1 overexpressed HeLamycTNKS1BP1 cells and control HeLa-NC cells with an inhibitor of DNA-PKcs (Nu7026), or an inhibitor of PARP-1 (3-AB), and an inhibitor of Tankyrase 1 (TNKS1) (XAV939), and used DMSO as control. As shown in Figure 5B, the phosphorylation of DNA-PKcs/ S2056 was largely blocked by Nu7026. The PARP-1 inhibitor 3-AB partially attenuated the TNKS1BP1mediated DNA-PKes phosphorylation at Ser2056. However, XAV939 displayed a relative weaker role as compared to the effect of 3-AB (Figure 5B). Moreover, phosphorylation of DNA-PKcs induced by ionizing radiation was also attenuated by the PARP-1 inhibitor 3-AB (Figure 5C). To further confirm the effect of PARP1 on TNKS1BP1-mediated DNA-PKcs phosphorylation, we performed the immunofluorescence assay. There was an increased phosphorylation of DNA-PKcs mediated by overexpressing HA-tagged TNKS1BP1, which was largely blocked by PARP inhibitor 3-AB (Figure 5D). Taken together, these results demonstrated that
TNKS1BP1 mediated the phosphorylation of DNA-PKcs at Ser2056, which could be attenuated by PARP inhibitors.

\section{DISCUSSION}

TNKS1BP1 was firstly identified as an interaction partner of tankyrase 1, and it can be poly(ADP-ribosyl) yated by tankyrase 1 in vitro and in vivo [32, 33]. Here, we demonstrated that TNKS1BP1 is involved in the IR-induced DNA damage response. An increased expression of TNKS1BP1 was firstly observed in the cells after irradiation. Depletion of TNKS1BP1 impaired the efficiency of DNA double-strand break repair and significantly increased the sensitivity of cells to IR. TNKS1BP1-deficient HeLa displayed a much higher level of residual $\gamma \mathrm{H} 2 \mathrm{AX}$ foci, a DSB biomarker, after certain times of post-irradiation repair as compared to TNKS1BP1-proficient HeLa cells, consistent with prolonged $\gamma \mathrm{H} 2 \mathrm{AX}$ kinetics. The defective DSB repair caused by depletion of TNKS1BP1 was also confirmed using comet and neutral pulsed-field gel electrophoresis assays. It has been reported that the localization of
A

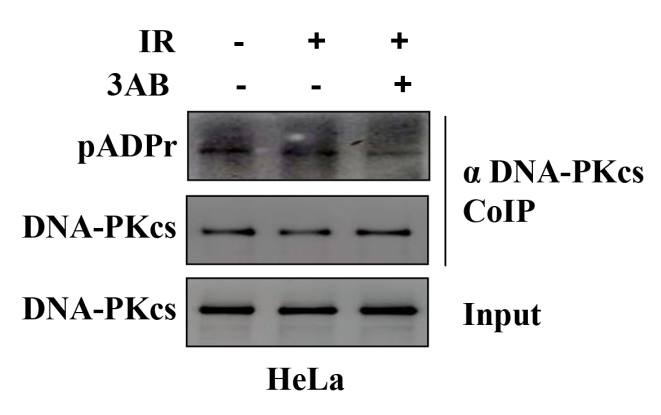

C

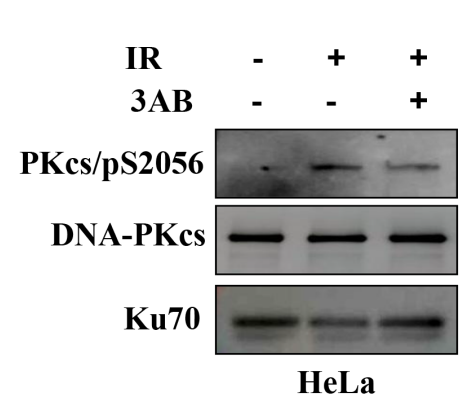

D
B
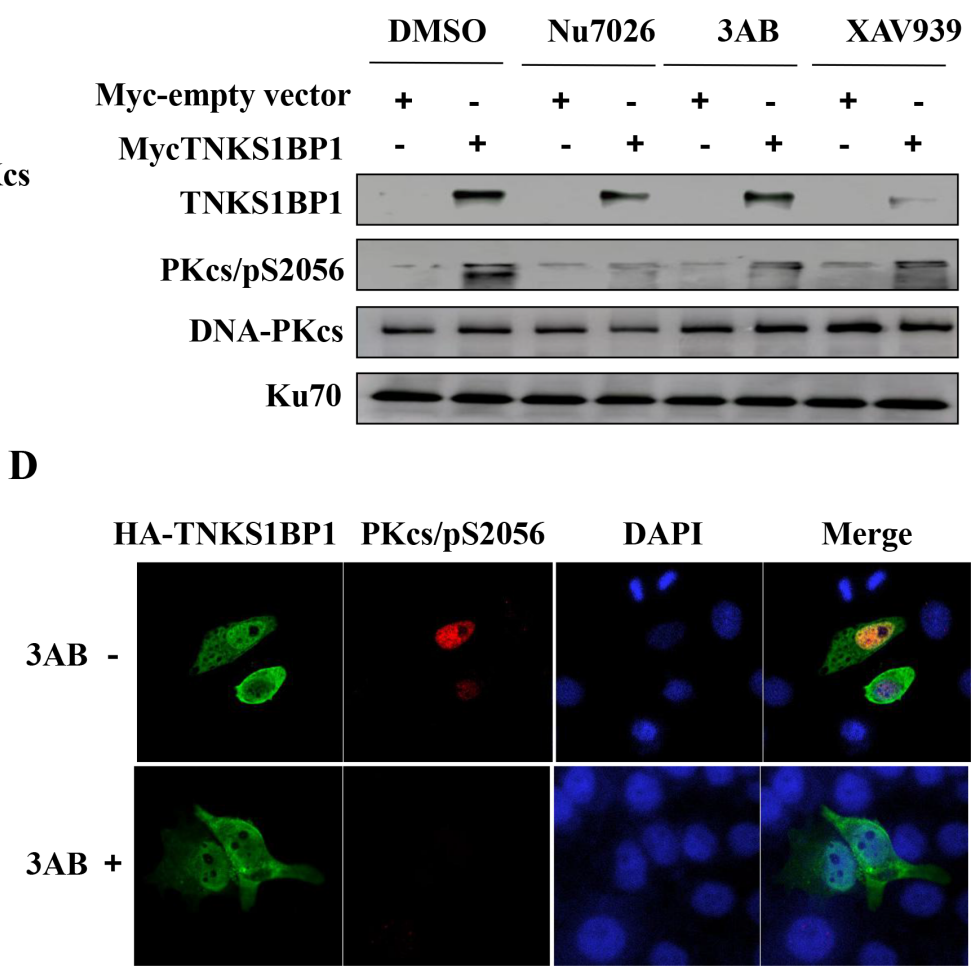

Figure 5: PARP-1 is responsible for TNKS1BP1-induced autophosphorlation of DNA-PKcs/S2056. (A) PARP-1 inhibitor 3-AB decreased the poly(ADP-ribosy)lation of DNA-PKcs. The Co-IP complex of the antibody against DNA-PKcs was separated by SDS-PAGE and immunoblotted with anti-pADPr antibody, anti-DNA-PKcs antibody. The immunoblotting of the input of cell lysates was used as sample control. (B) TNKS1BP1-induced autophosphorylation of DNA-PKcs/S2056 was attenuated by DNA-PKcs inhibitor Nu7056, PARP-1 inhibitor 3AB. The action of tankyrase 1 inhibitor XAV939 was relative weak. (C) PARP-1 inhibitor 3-AB decreased the autophosphorylation of DNA-PKcs/S2056. (D) Immunofluorescence image showing that the TNKS1BP1-induced autophosphrylation of DNA-PKcs/S2056 was blocked by 3-AB. HEK-293 cells were transfected with plasmid of HA-tagged TNKS1BP1, and subjected to co-immunofluorescence staining with DNA-PKcs/pS2056 antibody and HA antibody $48 \mathrm{~h}$ after plasmid transfection. 
TNKS1BP1 in cells exhibits a heterochromatic pattern in the nucleus. The large internal acidic region $(\mathrm{pI}=4.3)$ of TNKS1BP1 contributes an acidic protein with $\mathrm{pI}=$ 4.6, which may facilitate its association with chromatin through binding to basic chromatin proteins, such as histones [32]. Previous observations suggested that TNKS1BP1 associated with heterochromatin protein 1 (HP1) [32]. Therefore, we assumed that as a chromatin associating protein, TNKS1BP1 may play a role in the assembly of DNA damage response protein complex around the DNA damage site when DNA DSBs occur.

In response to DSBs, DNA-PKcs is phosphorylated at multiple sites, among which Ser2056 is a bona fide autophosphorylation site. The autophosphorylation of DNA-PKcs/S2056 can be induced by ionizing radiation and other DNA damage agents [4, 7, 37], and is necessary for the activation of the DSB NHEJ repair pathway. Our study demonstrated that overexpression of TNKS1BP1 effectively induced the autophosphorylation of DNA-PKcs/S2056. This TNKS1BP1-related autophosphorylation of DNA-PKcs was abolished by its kinase inhibitor Nu7026. However, TNKS1BP1 did not induce the phosphorylation of DNA-PKcs at the site of T2609 (data not shown). It appears that TNKS1BP1 plays a role in activating DNA-PKes through regulating its autophosphorylation.

In order to reveal the mechanism of TNKS1BP1 in regulating DNA-PKcs autophosphorylation, we identified the interacting proteins by co-immunoprecipitation assays using the antibody against TNKS1BP1/TAB182. Our study demonstrated that TNKS1BP1 interacted with the DNA-PKcs/Ku70/Ku86 complex and PARP-1. Although it has previously been reported that DNA-PKcs and PARP-1 are substrates for each other [24, 27], the purified DNA-PKcs and PARP-1 did not interact directly in vitro [24]. Therefore, their interaction in cells is believed to be mediated by other protein(s). Our study showed that depletion of TNKS1BP1 attenuated the association of DNA-PKcs and PARP-1, implying that TNKS1BP1 is responsible for mediating the interaction of DNA-PKcs and PARP-1.

PARP-1 is the most abundant member of the poly(ADP-ribose) polymerase superfamily in cells. PARP-1 has a carboxyl-terminal catalytic domain that polymerizes linear or branched chains of ADP-ribose (ADPR) from donor nicotinamide adenine dinucleotide $\left(\mathrm{NAD}^{+}\right)$onto the target proteins. Poly(ADP-ribosyl)ation of proteins, also called PARylation, by PARP-1 is one type of post-translation modifications, which is involved in regulating gene expression and DNA damage repair. It has been reported that DNA-PKes was PARylated by PARP-1 in HeLa cells after the stimulation of IFN- $\gamma$ [25], and this PARylation is required for the activation of DNA-PKcs. Here, we also detected the PARylation of DNA-PKcs, which was abolished by the PARP-1 inhibitor 3-AB. Similar to DNA-PKcs, PARP-1 can be activated by DNA strand breaks and is also a key component involved in DNA damage recognition, repair and signal transduction. Accumulated evidence suggested that PARP1 and DNA-PKcs cooperated within the same pathway to promote DSBs repair. Inhibition of PARP-1 by a specific inhibitor not only impaired the efficiency of single strand break repair and base excision repair, but also decreased the efficiency of the NHEJ pathway of DSB repair when cells were exposed to ionizing radiation $[21,26]$. Several PARP-1 inhibitors have been explored to cancer therapy either alone or in combination with radiotherapy or other DNA-damaging drugs [38, 39]. In addition, DNA-PKcs was shown overexpressed or highly activated in many tumors and its upregulation was correlated with advanced stage and poor patient prognosis [40-42]. A series of studies have demonstrated that targeting DNA-PKcs is an attractive strategy of cancer therapy [35, 42-44]. Recently, Verghese et al reported that the miR-26b was a highly deregulated microRNA in the carcinoma-associated fibroblasts (CAFs) in breast cancers, and the reduced miR$26 \mathrm{~b}$ expression caused an enhancement of the fibroblast migration and invasion. TNSK1BP1 was identified to be one of the direct downstream key targets of miR-26b. Moreover, the high expression of TNSK1BP1 in cancer stroma was significantly associated with the increased rates of breast cancer recurrence [45]. Therefore, all of these three molecules DNA-PKcs, PARP-1 and TNSK1BP1 are obviously implicated whether with the cancers prognosis or with the outcome of radio- or chemotherapy. Our results has provided novel information demonstrating that TNKS1BP1 functions as a bridge linking the PARP-1 and DNA-PKcs, a critical component of the NHEJ pathway of DNA DSB repair, to stimulate the autophosphorylation of DNA-PKcs in the cellular response to ionizing radiation-induced DNA double-strand breaks.

In summary, for the first time we demonstrated the biological function of TNSK1BP1 participating in the regulation of DNA DSB repair. TNKS1BP1 mediates the interaction of DNA-PKcs and PARP-1, which leads to activation of DNA-PKcs autophosphorylation. The PARylation of DNA-PKcs by PARP-1 appears to play a role in above process.

\section{MATERIALS AND METHODS}

\section{Plasmids, antibodies and reagents}

TNKS1BP1/TAB182 expression vector (PLPCMYC-TAB182) was kindly provided by Dr. Susan Smith (Kimmel Center for Biology and Medicine of the Skirball Institute, New York University School of Medicine). PCMV-HA-TNKS1BP1 vector was generated by polymerase chain reaction (PCR) cloning. shRNATNKS1BP1-U6/Hygromycin vectors and shRNA-NC-U6/ hygromycin vectors were purchased from Genepharma. To obtain the RNA interference resistant TNKS1BP1 
expression plasmid (PCMV-HA-shiTNKS1BP1), the region targeted by the TNKS1BP1 shRNA was changed to GGAGAGTTTCTCAAGTCGAGGGAGCGTGGA. Mutagenesis of TNKS1BP1 was done using the Q5 hot start High-fidelity DNA polymerase. DNA-PKcs Inhibitor (Nu7026), PARP inhibitors (3-AB, XAV939), Propidium Iodide (PI) and DAPI were purchased from Sigma. All antibodies were commercial products. Anti-actin, antiDNA-PKcs, anti-Ku86, anti-PARP-1, anti-TAB182 (TNKS1BP1), anti-Myc (9E10) and anti-HA were purchased from Santa Cruz. Anti-Ku70, anti-pADPr and anti-phospho-DNA-PKcs (Ser2056) were purchased from Abcam. Anti-Phospho-H2AX (Ser139) was purchased from Millipore, AlexaFlour 568-goat anti-rabbit IgG and AlexaFlour 488-goat anti-mouse IgG were purchased from Invitrogen.

\section{Cell lines, culture and ionizing radiation}

All cell lines were obtained from ATCC. HeLa human cervical cancer cell line, HepG2 human hepatocellular cancer cell line, MCF-7 human breast cancer cell line and human embryonic kidney HEK 293 cells were cultured in DMEM medium (Gibco) supplemented with 10\% FBS (Hygclone), and maintained in a humidified incubator with $5 \% \mathrm{CO}_{2}, 37^{\circ} \mathrm{C}$. HeLashTNKS1BP1 and HeLa-NC, HepG2-shTNKS1BP1 and HepG2-NC cells were generated from HeLa cells and HepG2 cells, respectively, via stably transfecting with shRNA-TNKS1BP1-U6/Hygromycin vectors targeting the TNKS1BP1 or a control shRNA-NC-U6/ hygromycin vector. A cell line with stable overexpression of TNKS1BP1 was generated by transfecting PLPCMYC-TNKS1BP1 or an empty vector (as a control) into $\mathrm{HeLa}$ cells. Colonies showing resistance to puromycin $(2-\mathrm{ug} / \mathrm{ml})$ were isolated and analyzed by immunoblotting. Plasmid transfection of HEK-293 cells was performed with Lipofectamine 2000 (Invitrogen) and the transfected HEK-293 cells were analyzed after $48 \mathrm{~h}$. Cells were irradiated using ${ }^{60} \mathrm{Co} \gamma$-rays at a dose rate of $1.98 \mathrm{~Gy} / \mathrm{min}$.

\section{Immunoblotting hybridization and immunofluorescent staining}

For immunoblotting (western blotting) analysis, cells with or without transfection of PLPC-MYCTNKS1BP1, PCMV-HA-TNKS1BP1 or PCMV-HAshiTNKS1BP1 plasmids for $48 \mathrm{~h}$, were given $4 \mathrm{~Gy}$ irradiation, and harvested at the indicated time points, and then lysed in the NETN lysis buffer $(100 \mathrm{mM} \mathrm{NaCl}, 20$ $\mathrm{mM}$ Tris-Cl (pH 8.0), $0.5 \mathrm{mM}$ EDTA, $0.5 \%$ (v/v) Nonidet P-40 (NP-40)) containing a cocktail of protease inhibitors (Roche). The nuclear and cytoplasmic extracts were prepared from HeLa cells given 4Gy or no irradiation, using a NE-PER kit (Thermo Fisher Scientific). Equal amounts of protein from each sample were injected into SDS-PAGE, then separated and transferred onto a Nitrocellulose membrane (Millipore). The blotting was blocked with $5 \%$ milk power in TBST $(20 \mathrm{mM}$ Tris-HCl, $500 \mathrm{mM} \mathrm{NaCl}(\mathrm{pH} 7.5), 0.1 \%(\mathrm{v} / \mathrm{v})$ Tween 20$)$ for $1 \mathrm{~h}$ at room temperature, then incubated overnight with the indicated antibody and washed with TBST. Bands were visualized by Imagequant LAS500 (GE).

For immunofluorescent staining, HeLa, HeLashTNKS1BP1 or HeLa-NC were transfected with TNKS1BP1 expression vector for $48 \mathrm{~h}$, and irradiated with a dose of $4 \mathrm{~Gy}$, then fixed in chilled phosphate-buffer saline (PBS) containing 4\% paraformaldehyde overnight at $4{ }^{\circ} \mathrm{C}$. The fixed cells were permeated in PBS containing $0.25 \%$ Trion X-100 for 30 min and washed with PBS for 3 times at room temperature, then blocked with $1 \%$ BSA in PBS for $30 \mathrm{~min}$. The cells were incubated with the indicated primary antibodies for $12 \mathrm{~h}$ at $4{ }^{\circ} \mathrm{C}$, washed with PBS for 3 times, incubated with AlexaFlour 568goat anti-rabbit IgG and AlexaFlour 488-goat anti-mouse IgG for $1 \mathrm{~h}$ at room temperature, washed with PBS for 3 times again, stained with DAP1 to visualize the DNA at room temperature, and observed using a LSM 510 laserscanning confocal microscope (Zeiss).

\section{RNA isolation and TNKS1BP1 mRNA expression analysis by quantitative real-time RT-PCR}

Total RNA was extracted from HeLa cells harvested at $2 \mathrm{~h}$ post 4 Gy using TRlzol reagent (Invitrogen) as recommended by the manufacturer. $1 \mu \mathrm{g}$ of total RNA was then used to reverse transcription using the ReverTra Ace qPCR RT Master Mix kit (Toyobo Life Science). Real-time PCR was performed for detecting TNKS1BP1 expression with a Bio-Rad iCycler \& iQ Real-time PCR systems (Bio-Rad) and a fluorescence-labeled SYBR Green real master Mix kit (TIANGEN Biotech (Beijing) Co. LTD.). Actin was used as an endogenous control. The sequence of forward and reverse primers for $T N K S 1 B P 1$ and actin are the following: TNKSIBP1 forward 5'-GTCAGGACTTCTCCTTCATTGAG-3' and reverse 5'-AGGCCAGGAAAGAGGTTGACTT-3'; actin forward 5'-CAGAGCAAGAGAGGCATCCT-3' and reverse 5'-TTGAAGGTCTCAAACATGAT-3'. Each sample was tested three times, and the expression levels of TNKS1BP1 were normalized to actin mRNA.

\section{Immunoprecipitation assay}

To detect the interaction complex of TNKS1BP1, DNA-PKcs, $\mathrm{Ku}$ and PARP-1, cells were lysed in a cold NTEN buffer (100 mM NaCl, $20 \mathrm{mM}$ Tris-Cl (pH 8.0), 0.5 mM EDTA, $0.5 \%$ (v/v) Nonidet P-40 (NP-40)) for $20 \mathrm{~min}$ on ice, then the lysates were centrifugated at $12000 \mathrm{~g}$ for $30 \mathrm{~min}$ at $4^{\circ} \mathrm{C}$. For immunoprecipitation, the supernatants were collected and mixed with $2 \mu \mathrm{g}$ of the indicated primary antibodies, and incubated for $12 \mathrm{~h}$ at $4{ }^{\circ} \mathrm{C}$, then incubated with protein $\mathrm{A} / \mathrm{G}$ agarose (Santa Cruz) for $4 \mathrm{~h}$ 
at $4^{\circ} \mathrm{C}$. Immunoprecipitated complexes were collected by centrifugation at $1000 \mathrm{~g}$ for $3 \mathrm{~min}$ at $4^{\circ} \mathrm{C}$, washed 4 times with NTEN buffer, and analyzed by western blotting hybridization.

\section{Colony formation assay}

Exponentially growing cells were collected and counted. Then the cells were irradiated at the different doses of $0,1,2,4,6$ Gy and plated into $60 \mathrm{~mm}$ dishes with a certain number of cells per dish, respectively. Cells were fed with DMEM medium supplemented with 10\% FBS for 2 weeks, then fixed with $70 \%$ ethanol, and stained with $1 \%$ crystal violet. The number of colonies containing more than 50 cells was calculated on each dish.

\section{Pulsed field gel electrophoresis (PFGE)}

For pulsed field gel electrophoresis (PFGE), after 20 Gy irradiation Hela-NC and HeLa-shTNKS1BP1 cells were harvested at the indicated time points, washed 3 times with PBS, resuspended with $50 \mu$ cell resuspension buffer (10 $\mathrm{mM}$ Tris, $\mathrm{pH} 7.2,20 \mathrm{mM} \mathrm{NaCl}$, $50 \mathrm{mM}$ EDTA). Then the cells were maintained at $50^{\circ} \mathrm{C}$ and mixed with $2 \% \operatorname{LMP}\left(50^{\circ} \mathrm{C}\right)$ at a ratio of $1: 1(\mathrm{v} / \mathrm{v})$. After being placed into a gel pore, the cell-agarose mixture was maintained at $4^{\circ} \mathrm{C}$ for solidifying, and digested in proteinase $\mathrm{K}$ reaction buffer (100 mM EDTA, pH 8.0, 0.2\% sodium deoxycholate, $1 \%$ sodium lauroyl sarcosine, $0.5 \mathrm{mg} / \mathrm{ml}$ proteinase $\mathrm{K}$ ) at $50^{\circ} \mathrm{C}$ for $24 \mathrm{~h}$. Then the cells/agarose mixture was washed 3 times in wash buffer (20 mM Tris, $\mathrm{pH} 8.0,50 \mathrm{mM}$ EDTA) gently at room temperature, and inserted into a $10 \%$ agarose gel and subjected to electrophoresis for $72 \mathrm{~h}$ under the conditions of $1.5 \mathrm{v} / \mathrm{cm}, 120$ degree of angle, $16^{\circ} \mathrm{C}$ of circulating buffer temperature. Thereafter the agarose gel was visualized using a gel imaging system and analyzed by Quantity One Soft.

\section{ACKNOWLEDGMENTS}

The Authors thank Professor Jolyon Hendry (Manchester University, UK) for helpful discussion and editorial assistance with the paper. We thank Dr Susan Smith (Kimmel Center for Biology and Medicine of the Skirbal Institute, New York University School of Medicine) for kindly providing the TNKS1BP1/TAB182 expression vector. This work was supported by the China National Science Foundation grant (Grant number: U1432248, 31370843, 81472919).

\section{DISCLOSURE OF POTENTIAL CONFLICTS OF INTEREST}

No potential conflicts of interest were disclosed.

\section{REFERENCES}

1. Zhou PK. DNA Damage, Signaling and Repair: Protecting genomic integrity and reducing the risk of human disease. Chinese Science Bulletin. 2011; 56:3119-3121.

2. Lieber MR. The mechanism of double-strand DNA break repair by the nonhomologous DNA end-joining pathway. Annu Rev Biochem. 2010; 79:181-211.

3. Davis AJ, Chen BP, Chen DJ. DNA-PK: a dynamic enzyme in a versatile DSB repair pathway. DNA Repair (Amst). 2014; 17:21-29.

4. Neal JA, Sugiman-Marangos S, VanderVere-Carozza P, Wagner M, Turchi J, Lees-Miller SP, Junop MS, Meek K. Unraveling the complexities of DNA-dependent protein kinase autophosphorylation. Molecular and Cellular Biology. 2014; 34:2162-2175.

5. Bakkenist CJ, Kastan MB. Initiating cellular stress responses. Cell. 2004; 118:9-17.

6. Abraham RT. PI -kinase related kinases: 'big' players in stress-induced signaling pathways. DNA Repair (Amst). 2004; 3:883-887.

7. Uematsu N, Weterings E, Yano K, Morotomi-Yano K, Jakob B, Taucher-Scholz G, Mari PO, van Gent DC, Chen BP, Chen DJ. Autophosphorylation of DNA-PKCS regulates its dynamics at DNA double-strand breaks. The Journal of Cell Biology. 2007; 177:219-229.

8. Chen BP, Uematsu N, Kobayashi J, Lerenthal Y, Krempler A, Yajima H, Lobrich M, Shiloh Y, Chen DJ. Ataxia telangiectasia mutated (ATM) is essential for DNAPKcs phosphorylations at the Thr-2609 cluster upon DNA double strand break. The Journal of Biological Chemistry. 2007; 282:6582-6587.

9. Davis AJ, Chen DJ. DNA double strand break repair via non-homologous end-joining. Translational Cancer Research. 2013; 2:130-143.

10. $\mathrm{Li} \mathrm{M}, \mathrm{Yu} \mathrm{X}$. The role of poly(ADP-ribosyl)ation in DNA damage response and cancer chemotherapy. Oncogene. 2014; Sep 15; [Epub ahead of print] Review.

11. Beck C, Robert I, Reina-San-Martin B, Schreiber V, Dantzer F. Poly(ADP-ribose) polymerases in doublestrand break repair: Focus on PARP1, PARP2 and PARP3. Experimental Cell Research. 2014; 329:18-25.

12. Krishnakumar R, Kraus WL. The PARP side of the nucleus: molecular actions, physiological outcomes, and clinical targets. Mol Cell. 2010; 39:8-24.

13. Langelier MF, Planck JL, Roy S, Pascal JM. Crystal structures of poly(ADP-ribose) polymerase-1 (PARP-1) zinc fingers bound to DNA: structural and functional insights into DNA-dependent PARP-1 activity. The Journal of Biological Chemistry. 2011; 286:10690-10701.

14. Langelier MF, Planck JL, Roy S, Pascal JM. Structural basis for DNA damage-dependent poly(ADP-ribosyl)ation by human PARP-1. Science. 2012; 336:728-732. 
15. Haince JF, McDonald D, Rodrigue A, Dery U, Masson JY, Hendzel MJ, Poirier GG. PARP1-dependent kinetics of recruitment of MRE11 and NBS1 proteins to multiple DNA damage sites. The Journal of Biological Chemistry. 2008; 283:1197-1208.

16. Li M, Yu X. Function of BRCA1 in the DNA damage response is mediated by ADP-ribosylation. Cancer Cell. 2013; 23:693-704.

17. Haince JF, Kozlov S, Dawson VL, Dawson TM, Hendzel MJ, Lavin MF, Poirier GG. Ataxia telangiectasia mutated (ATM) signaling network is modulated by a novel poly(ADP-ribose)-dependent pathway in the early response to DNA-damaging agents. The Journal of Biological Chemistry. 2007; 282:16441-16453.

18. Ahel D, Horejsi Z, Wiechens N, Polo SE, Garcia-Wilson E, Ahel I, Flynn H, Skehel M, West SC, Jackson SP, OwenHughes T, Boulton SJ. Poly(ADP-ribose)-dependent regulation of DNA repair by the chromatin remodeling enzyme ALC1. Science. 2009; 325:1240-1243.

19. Gagne JP, Isabelle M, Lo KS, Bourassa S, Hendzel MJ, Dawson VL, Dawson TM, Poirier GG. Proteome-wide identification of poly(ADP-ribose) binding proteins and poly(ADP-ribose)-associated protein complexes. Nucleic Acids Res. 2008; 36:6959-6976.

20. Gottschalk AJ, Timinszky G, Kong SE, Jin J, Cai Y, Swanson SK, Washburn MP, Florens L, Ladurner AG, Conaway JW, Conaway RC. Poly(ADP-ribosyl)ation directs recruitment and activation of an ATP-dependent chromatin remodeler. Proceedings of the National Academy of Sciences of the United States of America. 2009; 106:13770-13774.

21. Mitchell J, Smith GC, Curtin NJ. Poly(ADP-Ribose) polymerase-1 and DNA-dependent protein kinase have equivalent roles in double strand break repair following ionizing radiation. Int J Radiat Oncol Biol Phys. 2009; 75:1520-1527.

22. Veuger SJ, Curtin NJ, Richardson CJ, Smith GC, Durkacz BW. Radiosensitization and DNA repair inhibition by the combined use of novel inhibitors of DNA-dependent protein kinase and poly(ADP-ribose) polymerase-1. Cancer Research. 2003; 63:6008-6015.

23. Spagnolo L, Barbeau J, Curtin NJ, Morris EP, Pearl LH. Visualization of a DNA-PK/PARP1 complex. Nucleic Acids Res. 2012; 40:4168-4177.

24. Ruscetti T, Lehnert BE, Halbrook J, Le Trong H, Hoekstra MF, Chen DJ, Peterson SR. Stimulation of the DNA-dependent protein kinase by poly(ADP-ribose) polymerase. The Journal of Biological Chemistry. 1998; 273:14461-14467.

25. Sajish M, Zhou Q, Kishi S, Valdez DM Jr, Kapoor M, Guo M, Lee S, Kim S, Yang XL, Schimmel P. Trp-tRNA synthetase bridges DNA-PKes to PARP-1 to link IFNgamma and p53 signaling. Nature Chemical Biology. 2012; 8:547-554.
26. Veuger SJ, Curtin NJ, Smith GC, Durkacz BW. Effects of novel inhibitors of poly(ADP-ribose) polymerase-1 and the DNA-dependent protein kinase on enzyme activities and DNA repair. Oncogene. 2004; 23:7322-7329.

27. Ariumi Y, Masutani M, Copeland TD, Mimori T, Sugimura T, Shimotohno K, Ueda K, Hatanaka M, Noda M. Suppression of the poly(ADP-ribose) polymerase activity by DNA-dependent protein kinase in vitro. Oncogene. 1999; 18:4616-4625.

28. Dregalla RC, Zhou J, Idate RR, Battaglia CL, Liber HL, Bailey SM. Regulatory roles of tankyrase 1 at telomeres and in DNA repair: suppression of T-SCE and stabilization of DNA-PKcs. Aging. 2010; 2:691-708.

29. Boehler C, Gauthier LR, Mortusewicz O, Biard DS, Saliou JM, Bresson A, Sanglier-Cianferani S, Smith S, Schreiber V, Boussin F, Dantzer F. Poly(ADP-ribose) polymerase 3 (PARP3), a newcomer in cellular response to DNA damage and mitotic progression. Proceedings of the National Academy of Sciences of the United States of America. 2011; 108:2783-2788.

30. Waaler J, Machon O, Tumova L, Dinh H, Korinek V, Wilson SR, Paulsen JE, Pedersen NM, Eide TJ, Machonova O, Gradl D, Voronkov A, von Kries JP, Krauss S. A novel tankyrase inhibitor decreases canonical Wnt signaling in colon carcinoma cells and reduces tumor growth in conditional APC mutant mice. Cancer Research. 2012; 72:2822-2832.

31. Casas-Selves M, Kim J, Zhang Z, Helfrich BA, Gao D, Porter CC, Scarborough HA, Bunn PA Jr, Chan DC, Tan AC, DeGregori J. Tankyrase and the canonical Wnt pathway protect lung cancer cells from EGFR inhibition. Cancer Research. 2012; 72:4154-4164.

32. Seimiya H, Smith S. The telomeric poly(ADP-ribose) polymerase, tankyrase 1, contains multiple binding sites for telomeric repeat binding factor 1 (TRF1) and a novel acceptor, 182-kDa tankyrase-binding protein (TAB182). The Journal of Biological Chemistry. 2002; 277:14116-14126.

33. Sbodio JI, Chi NW. Identification of a tankyrase-binding motif shared by IRAP, TAB182, and human TRF1 but not mouse TRF1. NuMA contains this RXXPDG motif and is a novel tankyrase partner. The Journal of Biological Chemistry. 2002; 277:31887-31892.

34. Shang Z, Yu L, Lin YF, Matsunaga S, Shen CY, Chen BP. DNA-PKcs activates the Chk2-Brcal pathway during mitosis to ensure chromosomal stability. Oncogenesis. 2014; 3:e85.

35. Shang ZF, Huang B, Xu QZ, Zhang SM, Fan R, Liu XD, Wang Y, Zhou PK. Inactivation of DNA-dependent protein kinase leads to spindle disruption and mitotic catastrophe with attenuated checkpoint protein 2 Phosphorylation in response to DNA damage. Cancer Research. 2010; 70:3657-3666.

36. Tu WZ, Li B, Huang B, Wang Y, Liu XD, Guan H, Zhang SM, Tang Y, Rang WQ, Zhou PK. gammaH2AX foci formation in the absence of DNA damage: mitotic 
H2AX phosphorylation is mediated by the DNA-PKcs/ CHK2 pathway. FEBS Lett. 2013; 587:3437-3443.

37. Chen BP, Chan DW, Kobayashi J, Burma S, Asaithamby A, Morotomi-Yano K, Botvinick E, Qin J, Chen DJ. Cell cycle dependence of DNA-dependent protein kinase phosphorylation in response to DNA double strand breaks. The Journal of Biological Chemistry. 2005; 280:14709-14715.

38. van Vuurden DG, Hulleman E, Meijer OL, Wedekind LE, Kool M, Witt H, Vandertop PW, Wurdinger T, Noske DP, Kaspers GJ, Cloos J. PARP inhibition sensitizes childhood high grade glioma, medulloblastoma and ependymoma to radiation. Oncotarget. 2011; 2:984-996.

39. Bridges KA, Toniatti C, Buser CA, Liu H, Buchholz TA, Meyn RE. Niraparib (MK-4827), a novel poly(ADPRibose) polymerase inhibitor, radiosensitizes human lung and breast cancer cells. Oncotarget. 2014; 5:5076-5086.

40. Hsu FM, Zhang S, Chen BP. Role of DNA-dependent protein kinase catalytic subunit in cancer development and treatment. Translational Cancer Research. 2012; 1:22-34.

41. Yu ZJ, Sui JG, Ding YQ, Cao ZS, Zhou PK, Wu DC. Expression of DNA-PK in hepato- and cholangio-neoplasms and its significance. Chinese Journal of Hepatology. 2004; 12:652-655.

42. An J, Xu QZ, Sui JL, Bai B, Zhou PK. Downregulation of c-myc protein by siRNA-mediated silencing of DNAPKcs in HeLa cells. International Journal of Cancer Journal International du Cancer. 2005; 117:531-537.

43. Ciszewski WM, Tavecchio M, Dastych J, Curtin NJ. DNA-PK inhibition by NU7441 sensitizes breast cancer cells to ionizing radiation and doxorubicin. Breast Cancer Research and Treatment. 2014; 143:47-55.

44. Yu L, Tumati V, Tseng SF, Hsu FM, Kim DN, Hong D, Hsieh JT, Jacobs C, Kapur P, Saha D. DAB2IP regulates autophagy in prostate cancer in response to combined treatment of radiation and a DNA-PKcs inhibitor. Neoplasia. 2012; 14:1203-1212.

45. Verghese ET, Drury R, Green CA, Holliday DL, Lu X, Nash C, Speirs V, Thorne JL, Thygesen HH, Zougman A, Hull MA, Hanby AM, Hughes TA. MiR-26b is downregulated in carcinoma-associated fibroblasts from ER-positive breast cancers leading to enhanced cell migration and invasion. The Journal of Pathology. 2013; 231:388-399. 